\title{
Nivel de estrés en docentes universitarios
}

Stress level of university teachers

\author{
María Esther Barradas Alarcón \\ Facultad de Psicología U.V. \\ ebarradas@uv.mx \\ Perfecto Gabriel Trujillo Castro. \\ Instituto Tecnológico de Veracruz México. \\ tecnito2009@prodigy.net.mx \\ María de Lourdes Lidia Guzmán Ibáñez \\ Facultad de Psicología U.V. \\ oguzman@uv.mx \\ Javier López González \\ Facultad de Psicología U.V. \\ jalopez@uv.mx
}

\section{Resumen}

Esta investigación tuvo como objetivo identificar el nivel de estrés, en docentes del área de ciencias de la salud y del área de humanidades de educación superior región Veracruz. Orientación metodológica es cuantitativa y el nivel de estudio descriptivo. Instrumento utilizado fue el test Perfil de Estrés Kenneth M. Nowak. La población fue 54 docentes. Resultados, con una distribución del $100 \%$ para cada área, en nivel alto de estrés se tuvo un $58 \%$ en docentes del área de la salud y un $7 \%$ en docentes del área de humanidades, con estrés moderado manifestaron el $29 \%$ de docentes de ciencias de la salud y el $13 \%$ de docentes de humanidades mientras que no presentaron estrés solo el $4 \%$ de docenes del área de ciencias de la salud y el $80 \%$ de docentes del área de humanidades

Palabras claves: Nivel de estrés. 


\section{Abstract}

This research aimed to identify the stress level in teachers in the area of health sciences and the humanities in higher education Veracruz region. Methodological approach is quantitative and descriptive study level. The test instrument was Stress Profile Kenneth M. Nowak. The population was 54 teachers. Results with $100 \%$ distribution for each area , high stress level teachers $58 \%$ in the area of health and $7 \%$ in teaching the humanities, with moderate stress had said $29 \%$ of teachers health sciences and $13 \%$ of teachers of humanities showed no stress while only $4 \%$ of the area teachers health sciences and $80 \%$ of teachers in the humanities

Key words: stress level.

Fecha recepción: Agosto 2014

Fecha aceptación: Marzo 2015

\section{Introducción}

El estrés es un tema que actualmente es muy investigado, debido a que este, se ha convertido en un padecimiento muy habitual y frecuente en nuestros tiempos, y va en aumento, convirtiéndose en un problema hacia la salud.

La mayoría de los individuos padecen estrés, quizá como producto de ciertos cambios tanto tecnológicos, ambientales y sociales, en nuestra forma de vivir y trabajar, que resultan imposible de adaptarse.

Es así, el interés por el estrés laboral se ha convertido en algo muy importante, debido a que afecta el rendimiento y actividad en el trabajo, existiendo muchas fuentes de estrés como la elección de decisiones, las diversas responsabilidades, el desagrado de alguna condiciones o de la organización del trabajo y entre un sin fin de cargas laborales. 
Es indispensable, en el desarrollo de la vida profesional plena, identificar los signos del estrés y con esto de alguna manera, poder prevenirlos y realizar un plan de acción.

De igual manera resulta relevante, este padecimiento, ya que se relaciona con la satisfacción y productividad del trabajador, además de que se pretende mejorar la calidad de vida en el trabajo.

Otra razón, es la gran repercusión en el factor económico, puesto que mucho dinero se invierte a las enfermedades relacionadas con el estrés, y en el ámbito docente se ha notado un considerable incremento.

Por lo tanto, el objetivo de este estudio fue identificar el nivel de estrés, en docentes del área de ciencias de la salud y del área de humanidades de la Universidad Veracruzana, región Veracruz.

\section{Estrés}

El estrés y los efectos que desencadena tanto en el individuo como en la sociedad, ha sido un tema de gran importancia y del cual se desprenden innumerables publicaciones ya sean estas desde la perspectiva social, psicológica o de salud en general; desde que, en 1935 Seyle consideraba que existía relación entre ciertas enfermedades como hipertensión arterial y trastornos emocionales, y las consecuencias resultantes de un estado prolongado de estrés. Se ha dado una mayor importancia al estudio de este trastorno y las diversas relaciones que este sostiene con las problemáticas de salud que enfrenta la sociedad moderna.

El ritmo de vida actual y las exigencias a las que se enfrentan la gran mayoría de las personas genera un estado de desequilibrio psíquico y fisiológico que a su vez desencadena una serie de síntomas o padecimientos que afectan la salud y bienestar de los miembros de una sociedad y siendo mayor el impacto en sociedades donde las políticas de los modelos económicos exigen cada día una mayor producción para satisfacer las necesidades de la misma; esto impacta de manera considerable en la salud, ya que la presión a la cual se es sometido a un individuo promedio dentro de una sociedad moderna genera estados emocionales y de salud carecientes de equilibro, es decir, la población en general vive 
dentro de una sociedad que está llena de estímulos que generan estrés y que están inevitablemente presentes en cada dimensión en las que se desenvuelve cualquier individuo.

Uno de los ambientes en dónde los individuos están más expuestos a los estímulos estresores es sin duda en el trabajo, el ambiente laboral es uno de los ambientes en el cual los individuos dedican un tercio de su tiempo cada día e incluso en muchas ocasiones el tiempo llega a ser hasta de la mitad del día dedicada a la realización de sus labores, dicho lo anterior no sería de extrañar que fuese justamente dentro de este en donde se presenten las principales fuentes de presión mismas que desencadenan una serie de respuestas de estrés en los individuos.

El estrés laboral es actualmente uno de los temas que más se estudia ya que de acuerdo con las últimas investigaciones, este puede generar reacciones negativas respecto a la actividad, la motivación, el rendimiento en el trabajo, entre otros. Y que este trastorno puede manifestarse en cualquier individuo en cualquier área de trabajo, sin duda esto es algo que las empresas o instituciones tienen que prestar importancia, ya que dentro de cualquier organización el factor humano es el activo más importante dentro de la misma.

Haciendo referencia al ámbito central del estudio, la docencia ha sido tradicionalmente considerada como una de las profesiones más estresantes. Estudios como el de Feitler y Tolkar (1997), en la cual se muestran que un 16 por ciento de los catedráticos consideran su profesión como" muy estresante" o "extremadamente estresante". Justamente la manera en que los catedráticos valoran su actividad laboral como fuente de estrés, puede llevar hacia expectativas que generen una mayor vulnerabilidad a este trastorno.

De acuerdo con (Allen, 2002) quien define al estrés como la respuesta de adaptación con la mediación de características individuales y/o procesos psicológicos, y que es consecuencia de cualquier acción, situación o acontecimiento externo que inflige demandas físicas y/o psicológicas a las personas.

En la actualidad es de vital importancia que las empresas e instituciones, participen en programas que apunten hacia mejoras dentro del ambiente laboral, sean estas desde las áreas en donde los trabajadores realizan sus actividades, es decir, infraestructura que le 
permita al trabajador la realización de sus labores sin obstaculización o limitación que pudiera resultar de una falta de elementos básicos para la ejecución o aplicación de sus conocimientos y la conclusión de tareas; hasta la relación interpersonal que se mantiene día a día con jefes y compañeros, de esta manera se propiciaría un desarrollo dentro de la organización que se preocupa en mejorar su calidad de trabajo, la salud de sus trabajadores y su productividad.

Para ello las empresas o instituciones deberán tomar en cuenta que para generar un ambiente que ayude a la minimización de fuentes desencadenantes de estrés, atenderá en primer lugar procesos de evaluación objetiva que permita conocer las situaciones bajo las cuales los trabajadores se encuentran desarrollando sus labores dentro de la organización.

Arce Arnaes \& Cols (2001) realizan un listado sobre los diversos factores permanentes que pueden provocar estés en las personas.

\section{Factores permanentes:}

1.- Novedad de la situación: Refiere la experiencia previa que se ha mantenido con la situación. Esta experiencia no tiene por qué ser directa, si no que puede inferirse a partir de información indirecta. Por ello, son escasas las situaciones completamente nuevas, ya que es fácil encontrar relación de semejanza con situaciones conocidas. Una situación nueva puede resultar estresante cuando existe una asociación previa con daño o peligro, de esta manera puede convertirse dicha novedad en una amenaza, Además, una situación nueva es ambigua y requiere una inferencia para su interpretación. En cuanto sea mayor la inferencia requerida, mayor será el grado de amenaza e incertidumbre.

2.- Predictibilidad: Implica la posibilidad de aprendizaje de determinadas características ambientales. La experiencia se ha realizado principalmente con animales; las conclusiones apuntan al hecho de que la estimulación predecible genera menor estado de tensión que la que no es predecible.

Sin embargo extrapolar estos datos al ser humano no sería de todo rigorista, ya que se deja de lado la mediación cognitiva, decisiva a la hora de hacer una explicación de las diferencias individuales del estrés. 
3.- Incertidumbre ante el acontecimiento: Influencia que puede tenerse sobre la valoración de la probabilidad de ocurrencia de un acontecimiento. El término incertidumbre introduce la noción de probabilidad. Pero esta probabilidad surge de manera subjetiva en la que los procesos cognitivos influyen de manera decisiva.

4.- Afrontamiento de la incertidumbre: Las circunstancias naturales de incertidumbre máxima suelen ser muy estresantes y es necesario tener en cuenta en estos acontecimientos un importante proceso mediador.

\section{Factores temporales:}

1.- Inminencia: Se refiere al tiempo que interviene antes de la aparición de un acontecimiento. La disminución de la inminencia puede llevar consigo un proceso complejo de valoración. Al disponer de más tiempo, el individuo desarrollará diversas estrategias de afrontamiento que influirán en las posteriores reevaluaciones. (Gandara, 1998)

2.- Duración: refiere el periodo de tiempo durante el que tiene lugar un acontecimiento estresante. En principio se supone que a mayor duración, mayor estrés.

Sin embargo hay que tener en cuenta el concepto de habituación, que hace referencia a la disminución de las respuestas (conductuales o fisiológicas), pudiendo aparecer también como resultado del afrontamiento cognitivo: el sujeto aprende a afrontar o evitar el agente estresante. (Ibid)

3.- Incertidumbre temporal: se refiere al desconocimiento sobre el momento en que tendrá lugar un acontecimiento concreto. Será estresante solo en casos en los que existan señales de amenaza que informen sobre la inminencia del acontecimiento. (Gándara, 1998)

4.- Ambigüedad: Característico de la mayoría de los acontecimientos que se dan en la vida cotidiana. Cuando la información necesaria para la evaluación de una situación es poco clara o insuficiente, la configuración del entorno es ambigua. Cuanto mayor es la ambigüedad, más factores personales determinan la importancia de la situación para el individuo.

En la ambigüedad van a influir de una u otra manera todas las características antes citadas (Manassero, 2003) 
Se han mencionado diferentes clasificaciones de estímulos que pueden generar una respuesta de estrés, ya sea por su contenido, por su frecuencia o duración. Sin embargo, para saber si el organismo dará una respuesta de estrés no basta con determinar si una situación es o no estresante. En este esquema, queda un elemento importante por señalar: las características del propio sujeto que pueden determinar la vulnerabilidad del mismo a dichas situaciones. Dicho de otro modo, el identificar un estímulo como estresante o no, está íntimamente ligada a la respuesta que el propio individuo dé a dicho estimulo. A su vez, esa respuesta está unida a las características del sujeto, responsable de la relevancia y significado que se confiera a la situación. (Manassero, 2003)

\section{Metodología}

\section{Descripción del Método.}

Tipo de investigación. El estudio es de tipo descriptivo que de acuerdo a Hernández, Fernández y Baptista (2014), pretende establecer las características, los niveles de asociación, las formas de manifestación o los índices de ocurrencia del problema de investigación.

Método. Se realizó a través de una metodología cuantitativa; que según Hernández, Fernández y Baptista (2014) es el método que utiliza la recolección y el análisis de datos para contestar preguntas de investigación, confiando en la medición numérica, el conteo y frecuentemente en el uso de la estadística para establecer con exactitud patrones de comportamiento en una población.

Definición de variable.- El estrés es la respuesta de adaptación con la mediación de características individuales y/o procesos psicológicos, y que es consecuencia de cualquier acción, situación o acontecimiento externo que inflige demandas físicas y/o psicológicas a las personas. (Lazarus, R. 1981).

Instrumento.- el test "Perfil de Estrés" de Kenneth M. Nowack, el cual proporciona una amplia evaluación del estrés y del riesgo para la salud incluyendo factores que demuestran relación entre estrés y enfermedad. 
El instrumento consta de 123 reactivos, conformado por siete áreas; estrés, hábitos de salud, red de apoyo social, conducta tipo A, fuerza cognitiva, estilo de afrontamiento y bienestar psicológico; dividido en 15 sub-escalas relacionadas con el estrés y el riesgo de enfermedad. Las propiedades psicométricas del instrumento reportan confiabilidad por mitades de 0,89 y 0,91 en poblaciones de diferentes razas y nivel educativo. La homogeneidad de las escalas con confiabilidad test-retest muestran un rango de 0,51 a 0,92 alpha de Cronbach y los análisis factoriales las reportan con baja a moderada relación $(0,41$ a 0,75 eigen).

Objetivo de Investigación.- identificar el nivel de estrés, y técnicas de afrontamientos de docentes del área de ciencias de la salud y docentes del área de humanidades de la Universidad Veracruzana región Veracruz.

Población.- de la carrera de ciencias de la salud fueron 24 docentes del área de ciencias de la salud $(\mathrm{CdS})$ y 30 docentes del área de humanidades $(\mathrm{H})$ con un rango en edades de 30 a 70 años y con nivel educativo de maestría y doctorado.

\section{Análisis de los resultados}

\section{Figura 1}

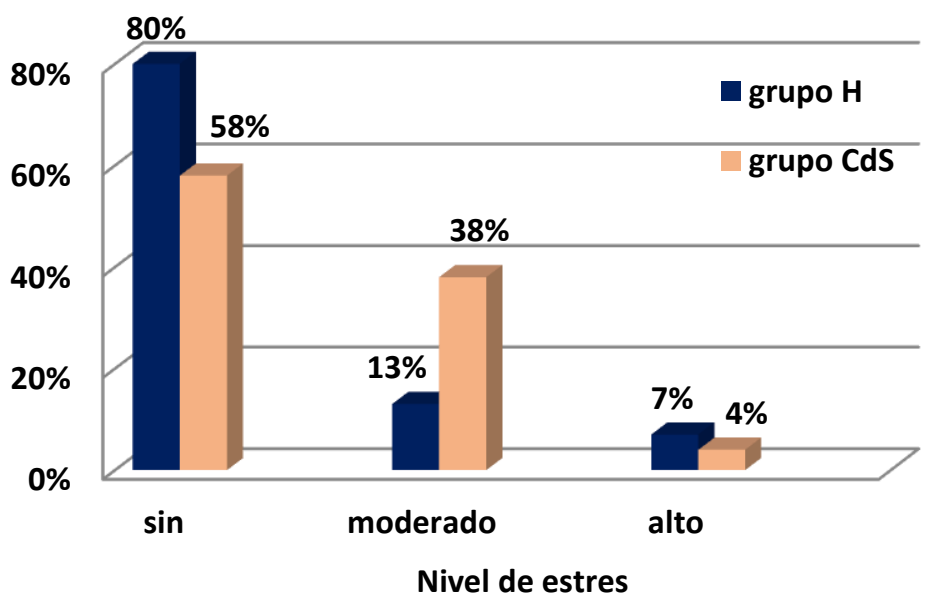


Fig 1 Nivel de estrés en Docentes del área de ciencias de la salud y del área de humanidades

a) Red de apoyo social.- Esta escala proporciona una medición directa del grado en el que el individuo siente que hay gente con la que puede contar en todo momento para obtener apoyo emocional, consejo, información, amor incondicional y ayuda, además de qué tan satisfecho se siente con dicho apoyo.

\section{Figura 2}

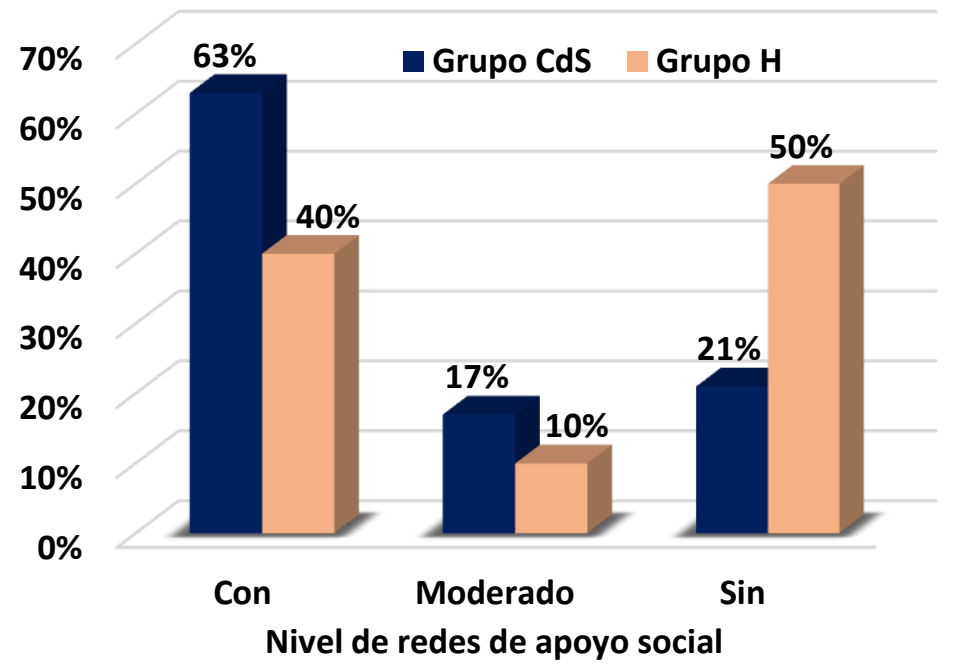

Fig. 2 Nivel de Redes de Apoyo con lo que cuentan los Docentes del área de ciencias de la salud y del área de humanidades

Los hábitos de salud son conductas específicas que cuando se practican de manera regular, conduce tanto al bienestar físico como psicológico. La escala se compone por reactivos que se agrupan en cuatro áreas principales, cada una de las cuales constituye una subescala: Ejercicio, descanso. Sueño, alimentación-nutrición y prevención. 


\section{Figura 3}

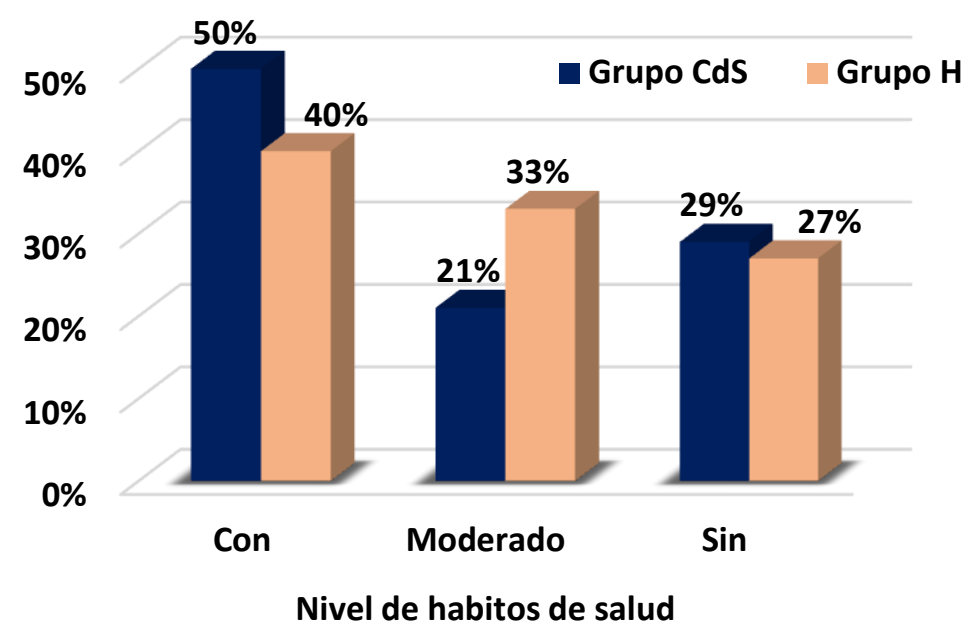

Fig. 3 Nivel de hábitos de salud en los Docentes del área de ciencias de la salud y del área de humanidades

En cuanto a los factores que integran los hábitos de salud tenemos la siguiente descripción:

- Ejercicio.- En esta subescalas se mide el nivel y la frecuencia del ejercicio que el individuo practica de manera regular para mejor el tono muscular y el sistema cardiovascular,

- Descanso-sueño: Aquí se explora la frecuencia con la que el individuo obtiene descanso adecuado, sueño y relajamiento.

- Alimentación-nutrición.-En estos reactivos se valora si la persona tiene una práctica general comer de manera balanceada y nutritiva.

- Prevención.- Aquí se mide la frecuencia con la que el individuo pone en práctica estrategias preventivas de salud e higiene. Además, se explora el uso de sustancias que están asociadas con una incidencia alta de problemas médicos. 


\section{Tabla I}

\begin{tabular}{|c|c|c|c|c|c|c|c|c|}
\hline \multicolumn{9}{|c|}{$\begin{array}{l}\text { Frecuencia (\%) de Hábitos de Salud con test Perfil de Estrés Kenneth } M \text {. Nowak en Académicos } \\
\text { de ciencias de salud y académicos de humanidades } \\
\qquad N=54\end{array}$} \\
\hline & \multicolumn{4}{|c|}{ EJERCICIO } & \multicolumn{4}{|c|}{ DESCANSO } \\
\hline & \multicolumn{2}{|c|}{$\begin{array}{l}\text { GRUPO CdS } \\
n(n=24) \%\end{array}$} & \multicolumn{2}{|c|}{$\begin{array}{r}\text { GRUPO H } \\
n \quad(n=30) \%\end{array}$} & \multicolumn{2}{|c|}{$\begin{array}{l}\text { GRUPO CdS } \\
n \quad(n=24) \%\end{array}$} & \multicolumn{2}{|c|}{$\begin{array}{c}\text { GRUPO H } \\
n \quad(n=30) \%\end{array}$} \\
\hline ALTO & 3 & 13 & 2 & 7 & 2 & 8 & 1 & 3 \\
\hline MODERADO & 9 & 38 & 8 & 37 & 8 & 33 & 20 & 67 \\
\hline \multirow[t]{3}{*}{$S I N$} & 12 & 50 & 14 & 57 & 14 & 58 & 9 & 30 \\
\hline & \multicolumn{4}{|c|}{ ALIMENTACIÓN } & \multicolumn{4}{|c|}{ PREVENCIÓN } \\
\hline & \multicolumn{2}{|c|}{$\begin{array}{l}\text { GRUPO CdS } \\
n(n=24) \%\end{array}$} & \multicolumn{2}{|c|}{$\begin{array}{c}\text { GRUPO H } \\
n(n=30) \%\end{array}$} & \multicolumn{2}{|c|}{$\begin{array}{r}\text { GRUPO CdS } \\
n(n=24) \%\end{array}$} & \multicolumn{2}{|c|}{$\begin{array}{r}\text { GRUPO H } \\
n \quad(n=30) \%\end{array}$} \\
\hline ALTO & 9 & 38 & 14 & 47 & 6 & 25 & 12 & 40 \\
\hline MODERADO & 8 & 33 & 8 & 27 & $\overline{11}$ & 46 & 10 & 33 \\
\hline SIN & 7 & 29 & 8 & 27 & 7 & 29 & 8 & 27 \\
\hline
\end{tabular}

Fuente: propia

a) En cuanto a la Conducta tipo A.- Mide el rango completo de las respuestas Tipo A expresadas, inclusive la ira internalizada, la ira expresada, premura de tiempo, rapidez laboral, impaciencia, involucramiento en el trabajo, búsqueda de mejoría, conducción ruda de vehículos y conductas competitivas. 


\section{Figura 4}

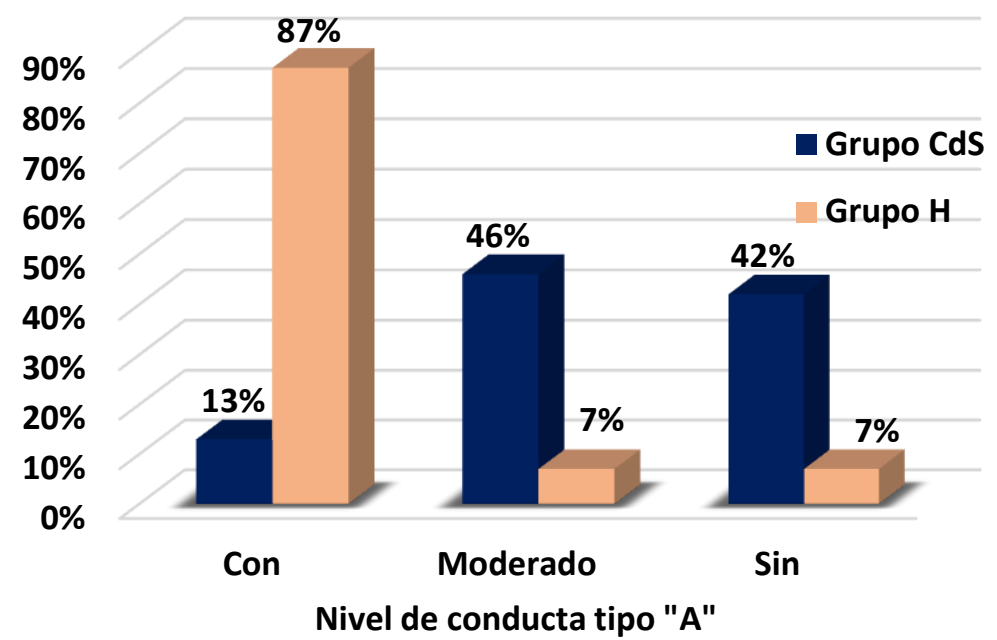

Fig. 4 Nivel de Conducta Tipo A en los Docentes del área de ciencias de la salud y del área de humanidades

a) Fuerzas cognitiva.- Explora las atribuciones, actitudes y creencias que un individuo tiene acerca de la vida y el trabajo.

Figura 5

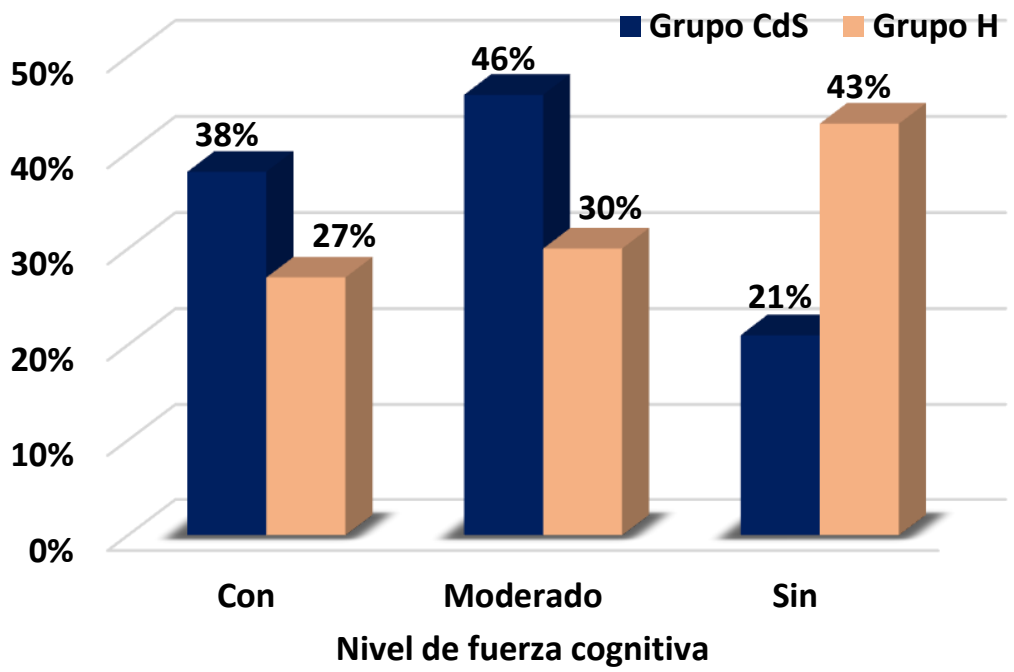

Fig. 5 Nivel de Fuerza Cognitiva en los Docentes del área de ciencias de la salud y del área de humanidades 
Con respecto a los estilos de afrontamiento.-Valora cuatro estrategias de afrontamiento que tienen una base conceptual diferente: Valoración positiva, Valoración negativa, Minimización de la amenaza y Concentración en el problema.

\section{Tabla II}

\begin{tabular}{|c|c|c|c|c|c|c|c|c|}
\hline \multicolumn{9}{|c|}{$\begin{array}{c}\text { Frecuencia (\%) de Técnicas de Afrontamiento con test Perfil de Estrés Kenneth } M \text {. Nowak er } \\
\text { Académicos de ciencias de salud y académicos de humanidades } \\
N=54\end{array}$} \\
\hline & \multicolumn{4}{|c|}{ VALORACION POSITIVA } & \multicolumn{4}{|c|}{ VALORACION NEGATIVA } \\
\hline & \multicolumn{2}{|c|}{$\begin{array}{l}\text { GRUPO CdS } \\
n(n=24) \%\end{array}$} & \multicolumn{2}{|c|}{$\begin{array}{r}\text { GRUPO H } \\
n \quad(n=30) \%\end{array}$} & \multicolumn{2}{|c|}{$\begin{array}{l}\text { GRUPO CdS } \\
n(n=24) \%\end{array}$} & \multicolumn{2}{|c|}{$\begin{array}{l}\text { GRUPO H } \\
n \quad(n=30) \%\end{array}$} \\
\hline ALTO & 9 & 38 & 13 & 43 & 4 & 17 & 22 & 73 \\
\hline MODERADO & 11 & 46 & 7 & 23 & 8 & 33 & 5 & 17 \\
\hline SIN & 4 & 17 & 10 & 33 & 12 & 50 & 3 & 10 \\
\hline & \multicolumn{4}{|c|}{ MINIMIZACION DE LA AMENAZA } & \multicolumn{4}{|c|}{$\frac{\text { CONCENTRACION DEL }}{\text { PROBLEMA }}$} \\
\hline & \multicolumn{2}{|c|}{$\begin{array}{l}\text { GRUPO CdS } \\
n(n=24) \%\end{array}$} & \multicolumn{2}{|c|}{$\begin{array}{r}\text { GRUPO H } \\
n \quad(n=30) \%\end{array}$} & \multicolumn{2}{|c|}{$\begin{array}{r}\text { GRUPO CdS } \\
n \quad(n=24) \%\end{array}$} & \multicolumn{2}{|c|}{$\begin{array}{r}\text { GRUPO H } \\
n \quad(n=30) \%\end{array}$} \\
\hline ALTO & 0 & 0 & 17 & 57 & 1 & 4 & 17 & 57 \\
\hline MODERADO & 14 & 58 & 7 & 23 & 11 & 46 & 10 & 33 \\
\hline SIN & 10 & 42 & 17 & 57 & 12 & 50 & 3 & 10 \\
\hline
\end{tabular}

Fuente: propia

- Valoración positiva.- Explora el uso de comentarios de apoyo y motivación autodirigido para minimizar la percepción del estrés.

- Valoración negativa.-Mide la tendencia a aproximarse a las situaciones desafiantes desde esta perspectiva.

- Minimización de la amenaza.- Explora la tendencia del individuo de afrontar los estresores mediante la mitigación de su significación o no extenderse en ellos. 
- Concentración en el problema.-En esta escala se indaga sobre la inclinación del sujeto a hacer intentos activos de cambiar su conducta o los estresores ambientales.

Con respecto al Conglomerado de reactivos ARC/ uso del alcohol, drogas recreativas y cigarrillos

\section{Figura 6}

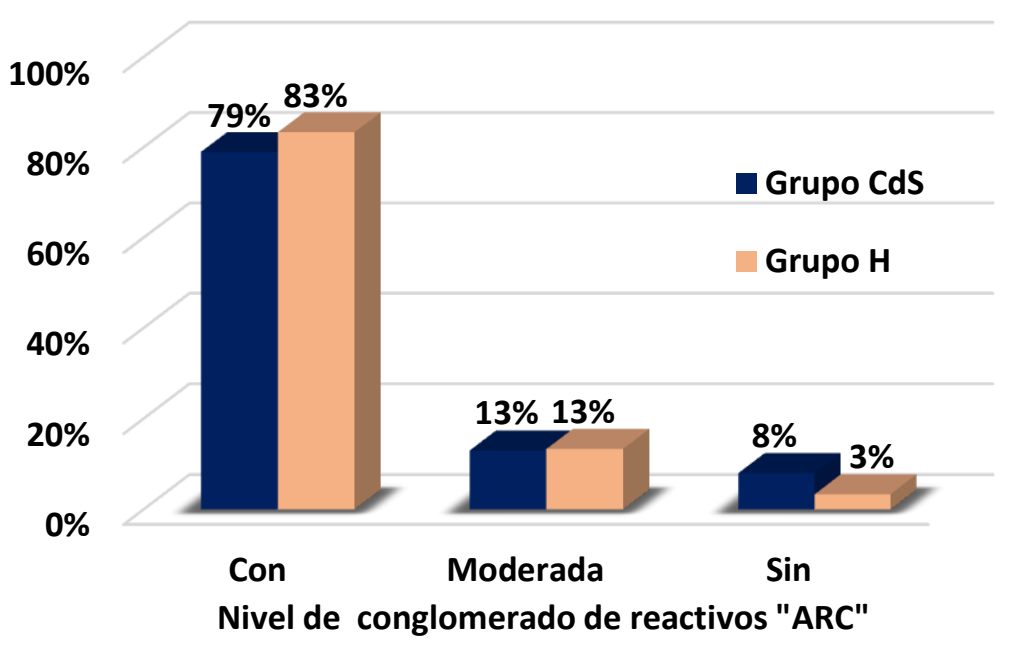

Fig. 6 Conglomerado de reactivos $\mathrm{ARC} /$ uso del alcohol, drogas recreativas y cigarrillos

En cuanto al Bienestar psicológico.- Valora la experiencia global del individuo de satisfacción y ecuanimidad psicológica durante los últimos tres meses. 


\section{Figura 7}

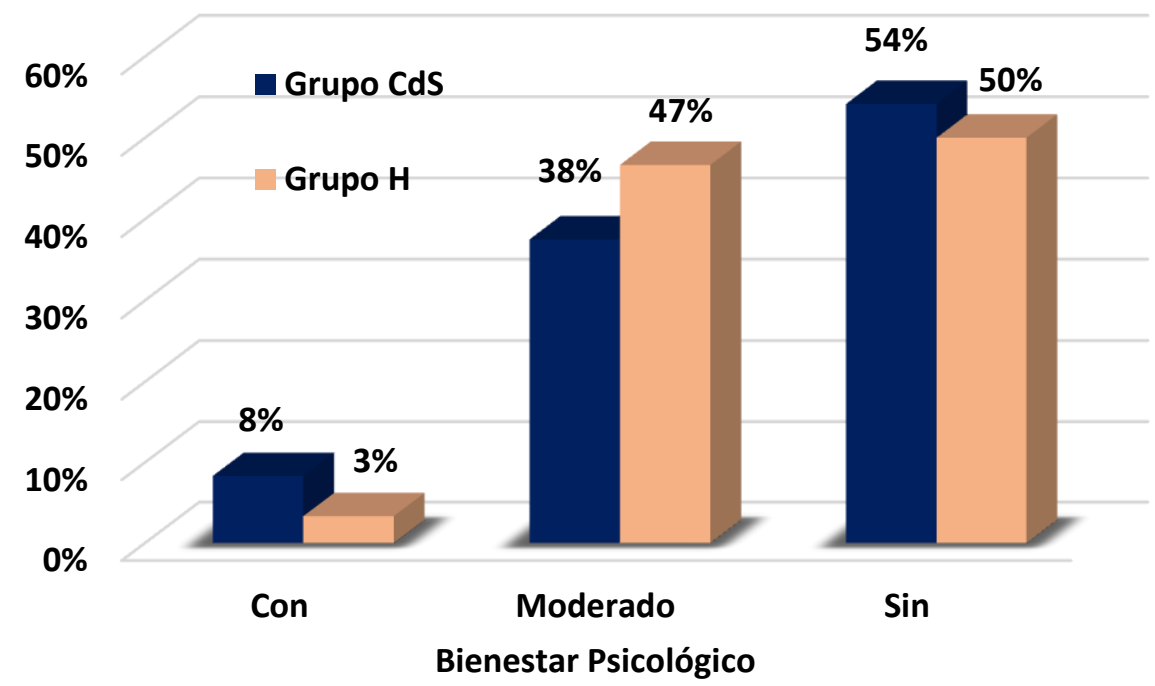

Fig. 7 Nivel de Bienestar Psicológico en los Docentes del área de ciencias de la salud y del área de humanidades

\section{Conclusiones}

De acuerdo a los resultados obtenidos con el test "Perfil de Estrés" se realizan las siguientes conclusiones por cada escala de evaluación.

Con una distribución del 100\% de los docentes para cada área la de ciencias de la salud, y de humanidades.

Estrés*: Tenemos que en cuanto al nivel de estrés alto los docentes del área de la salud manifestaron el $58 \%$ y solo el $7 \%$ en docentes de humanidades. Con respecto nivel de estrés moderado, el $29 \%$ lo obtuvo docentes de ciencias de la salud y el 13\% de docentes de humanidades finalmente el $4 \%$ de docenes del área de ciencias de la salud y el $80 \%$ de docentes del área de humanidades no presentaron estrés.

En una investigación realizada con catedráticos del Centro de Estudios Veracruz (CEV) campus Boca del Río. Parra Sánchez Abraham Cuauhtémoc (2009). El nivel de estrés global individual es del $80 \%$ de la población estudiada (30\% hombres y $50 \%$ mujeres) se encontró un nivel de estrés bajo, mientras que el 20\% (todos hombres) restante presentaron 
un nivel de estrés moderado; en lo que respecta al nivel de estrés global de grupo y/u organizacional, el $90 \%$ de la población estudiada (50\% mujeres y $40 \%$ hombres) se encuentran en un nivel de estrés bajo, mientras que el 10\% (hombres) restantes mostró un nivel de estrés moderado.

En otra investigación sobre estrés laboral y la sintomatología del estrés en el personal de enfermería de un hospital de segundo nivel del IMSS Benito Coquet Lagunes zona 71 de la ciudad de Veracruz ver”. Pérez Grajales Ada del Carmen (2009) Expone lo siguientes resultados el $54.8 \%$ manifiesta estrés laboral y el $45.2 \%$ no presenta este indicador.

Red de apoyo social: , Se tiene que el 10\% de los docentes de ciencias de la salud y el $63 \%$ de humanidades mencionan manifiestan sentimiento de abandono al no contar con apoyo emocional o ayuda por parte de amistades, pareja o familia, mientras que el el 10\% de docentes de ciencias de la salud y el 17\% de humanidades dicen que es moderad su red de apoyo y el $80 \%$ de docentes de ciencias de la salud y $20 \%$ de humanidades tienen un nivel alto de red apoyo es decir se muestran satisfechos al sentirse apoyados por las personas, esto puede ayudar a que experimenten seguridad en cada dimensión de su vida. Véase figura 2

> Hábitos de Salud: El tiene que el 50\% de docentes de ciencias de la saludo y el $40 \%$ de humanidades si presentan buenos hábitos de salud, significa que mantienen un estilo de vida que satisface los estándares generales aceptados para mantener la salud el $21 \%$ de docentes de ciencias de salud y el $33 \%$ de humanidades presentan moderados hábitos de salud, quiere decir que practican conductas orientadas al mantenimiento de la salud de manera regular y finalmente el $29 \%$ de docentes de ciencias de a salud y el $27 \%$ de humanidades No presentan hábitos de salud es decir reflejan un riesgo para la salud. Véase figura 3

D Ejercicio: El 50\% de docentes de ciencias de la salud y el 57\% de humanidades de la población no realiza actividades físicas regularmente, las cuales ayudan a disminuir la tensión y a prevenir el padecimiento de enfermedades cardiovasculares, además de fortalecer las redes de apoyo social mediante los ejercicios en grupo, Apenas el $38 \%$ de docentes de ciencias de la salud y el $37 \%$ de humanidades si 
realizan actividades físicas con una regularidad de dos a tres veces por semana durante 20 a 30 minutos. Y solo el 13\% de docentes de ciencias de la salud y el $7 \%$ de humanidades si practican el ejercicio físico. Véase Tabla I

Descanso/ Sueño: En esta sub escala arrojó un el 58\% de docentes de ciencias de la salud y el $30 \%$ de humanidades que no mantienen un horario regular para el descanso, el cual podría ser un riesgo para satisfacer las exigencias laborales, sociales y personales. Solo el 8,33\% mantienen un horario regular para satisfacer esta necesidad. Véase Tabla I

$>$ Alimentación/Nutrición: El el 33\% de docentes de ciencias de la salud y el 27\% de humanidades presentan hábitos alimenticios regulares, el 38\% de docentes de ciencias de la saludo y el $47 \%$ de humanidades tienden a mantener hábitos dietéticos balanceados, mientras que el el $29 \%$ de docentes de ciencias de la salud y el $27 \%$ de humanidades manifiesta riesgos de salud en sus hábitos de alimentación. Véase Tabla I

> Prevención: el 25\% de docentes de ciencias de la salud y el $40 \%$ de humanidades ponen en práctica estrategias preventivas de salud e higiene, mientras que el $29 \%$ de docentes de ciencias de la salud y el $27 \%$ de humanidades muestra vulnerabilidad ante o riesgo ante enfermedad.

$>$ Conducta tipo $\mathbf{A}^{*}$ : que el $13 \%$ de docentes de ciencias de la salud y el $87 \%$ de humanidades expresan conductas de tipo A con mayor frecuencia; posiblemente debido a su autoexigencia para mantener su nivel académico; lo que podría ser propicio para desarrollar diferentes enfermedades por lo que se considera un riesgo muy importante para la salud, específicamente en las enfermedades cardiacas. mientras que el $46 \%$ de docentes de ciencias de la salud y el $7 \%$ de humanidades son menos autoexigentes y competitivos, además se enojan con dificultad y se comportan de manera afectuosa y amable. finalmente el $42 \%$ de docentes de ciencias de a salud y el $7 \%$ de humanidades No presentan conducta tipo A. Véase figura 4

Fuerza cognitiva: Poco más del el $38 \%$ de los docentes de ciencias de la salud y el $27 \%$ de humanidades mencionan tienen Fuerzas cognitiva experimentan niveles 
altos de compromiso y participación en su trabajo, familia y consigo mismos, estás personas consideran los cambios de vida y los riesgo como una oportunidad de crecimiento personal y emocional. Mientras que el $46 \%$ de docentes de ciencias de la salud y el $30 \%$ de humanidades dicen que es moderada su fuerzas cognitiva y el $21 \%$ de docentes de ciencias de la salud el $43 \%$ de humanidades dicen no tener fuerzas cognitiva es decir una sensación de pérdida de control respecto los acontecimientos, no sobre los aspectos de importancia en su vida, esto último representa un riesgo para la salud. Véase figura 5

> Conglomerado ACR*: el 46\% de docentes de ciencias de la salud y el $83 \%$ de humanidades se mantienen fuera de riesgo frente al consumo de (Alcohol, drogas o tabaco), el $50 \%$ de docentes de ciencias de la salud y el $14 \%$ de humanidades tienen un riesgo moderado y el $7 \%$ de docentes de ciencias de la salud y el $3 \%$ de humanidades manifiesta un riesgo a la salud por consumo. Ver figura 6

\section{En cuanto a las técnicas de afrontamiento}

$>$ Valorización positiva: El el 38\% de docentes de ciencias de la saludo y el $43 \%$ de humanidades manifiestan un alto nivel en valoración positiva es decir los docentes emplean estrategias de afrontamiento concentrándose en aspectos positivos de las situaciones que se presentan ante sí como estresantes, esto supone una automotivación para minimizar la percepción del estrés. el 46\% de docentes de ciencias de la salud y el $23 \%$ de humanidades tienen utilizan de manera moderada la estrategia de fuerza positiva, finalmente el $17 \%$ de docentes de ciencias de la salud y el $33 \%$ de humanidades señalan No tener o utilizar la técnica de afrontamiento de valor positiva. Véase Tabla I

Valorización negativa*: En esta escala solo el $17 \%$ de docentes de ciencias de la saludo y el 73\% de humanidades consideran Manifestar un alto nivel en valoración negativa, el $33 \%$ de docentes de ciencias de la salud y el 17\% de humanidades tienen utilizan de manera moderada la estrategia de valoración negativa, se concentran mayormente en los peores aspectos o consecuencias de una situación, el $50 \%$ de docentes de ciencias de la salud y el $10 \%$ de humanidades señalan No tener o utilizar la técnica de afrontamiento de valoración negativa. Véase Tabla I 
Minimización de la amenaza: Esta escala muestra el 0\% de docentes de ciencias de la salud y el $57 \%$ de humanidades consideran utilizar la estrategia de minimización de la amenaza, el $58 \%$ de docentes de ciencias de la salud y el $23 \%$ de humanidades tienden utilizan de manera moderada la estrategia de minimización de la amenaza. Esta evitación hacia las situaciones problemáticas, podría ser útil para el individuo en casos dónde no se ha manifestado algún tipo de problemáticas objetivas en su salud, es decir, en los casos de padecimientos crónicos esta estrategia podría conducir a un deterioro en la salud y conducir a la muerte. finalmente el $42 \%$ de docentes de ciencias de la salud y el $57 \%$ de humanidades señalan No tener o utilizar la técnica de afrontamiento de minimización de la amenaza. Véase Tabla I

$>$ Concentración en el problema: el $4 \%$ de docentes de ciencias de la saludo y el $57 \%$ de humanidades consideran utilizar la estrategia de concentración del problema , el $46 \%$ de docentes de ciencias de la salud y el $33 \%$ de humanidades tienden a utilizan de manera moderada la estrategia de concentración del problema es decir muestran una inclinación a desarrollar intentos de cambios en su manera de actuar, desarrollando planes específicos de acción para dar solución a un conflicto analizando su propia experiencia o la de los demás para afrontar la situación estresante. , finalmente el $50 \%$ de docentes de ciencias de la salud y el $10 \%$ de humanidades señalan No tener o utilizar la técnica de afrontamiento de concentración del problema. Véase Tabla I

Bienestar Psicológico: se tiene que el $8 \%$ de los docentes de ciencias de la salud y el 3\% de humanidades expresan satisfacción general y disfrutan de su vida, considerándose llenos de felicidad. Solo el 8,33\% el 38\% de docentes de ciencias de la salud y el $47 \%$ de humanidades dicen que es moderad Bienestar psicológico y el $54 \%$ de docentes de ciencias de la salud el $50 \%$ de humanidades solo fue no tienen moderad Bienestar psicológico experimentan falta generalizada de bienestar en su vida, esto manifiesta un riesgo hacia su salud. . Véase figura 5

Finalmente como reflexión ejercer la docencia a nivel de educación superior es entrar sin buscarlo a una dinámica que de manera gradual va tomando una velocidad vertiginosa y un incremento de tareas, en las que de repente uno se pregunta ¿cómo 
llegue a esto?, pues la docencia en este caso para este estudio a nivel superior exige un mayor involucramiento en diversas tareas entre ellas, él que de manera permanente y continua hay que estarse actualizando, y paralelamente a impartir las clases, el realizar gestión, vinculación, investigación, realizar publicación para garantizar la permanencia laboral y a posibilidad a aspirar a un tiempo completo y con esto poder lograr un poco más la obtención de más recursos económicos. Lamentablemente estas condiciones laborales traen como consecuencia alteraciones consideradas en la calidad de vida de sus catedráticos. Ya que el incremento en las tareas les exponen al incremento de estrés dejándoles vulnerables a enfermedades físicas como emocionales entre ellos trastornos del sueño, trastornos digestivos como colitis, gastritis, cefaleas, contracturas, ansiedad, depresión, fatiga física, disfonías, parálisis facial, aun cáncer (Sánchez y Martínez, 2014).

Sugerencias. Aprender a decir "no", no ser perfeccionista, aceptar lo inevitable, reconsiderar los "debería..." de su vida, tomar conciencia de que no puede cambiar a los demás, sólo a usted mismo, Seleccionar la información para evitar saturarse,, no magnificar ni subestimar los problemas, aceptar que no es posible tener todo lo que se desea, aprender a identificar lo realmente importante, saber perdonarse a sí mismo y a los demás, ver las situaciones críticas como una oportunidad.

\section{Bibliografía}

Furnham, Adrián (2009) Psicología Organizacional: el comportamiento del individuo en las organizaciones. México. Editorial Alfa omega.

Hernández, S.R, Fernández C.F, Baptista L.P. (2010) Metodología de la investigación. México. Editorial Mc Graw Hill Quinta edición.

Ivancevich, John M (1989) Estrés y trabajo: una perspectiva gerencial 2da edición. México: editorial trillas. 
José María Peiró Silla (1999) Desencadenantes del estrés laboral. España. Ediciones Pirámide.

Lacan. J (1992) El seminario de Jaques Lacan. Libro 16 "De un otro al otro". Barcelona España. Editorial Paidós Ibérica, S.A.

Robbins. S, Timothy A. (2005) Comportamiento Organizacional. México. Editorial Pearson, $13^{\circ}$ edición.

Sánchez, C., y Martínez, S. (2014). "Condiciones de trabajo de docentes universitarios, satisfacción, exigencias laborales y daños a la salud", en: Salud de los trabajadores, Vol. 22(1), enero-junio, México, pp. 19-28.

Santiago, J; Tornay, F; Gómez, E. (1999) Procesos Psicológicos Básicos. España. Editorial Mc Graw Hill.

Stephen W, Cooper L (2004) Manejo del estrés en el trabajo. Plan de acción detallado para profesionales. México. Editorial: Manual Moderno.

Vygotsky, L. S. (1978). Pensamiento y lenguaje. Madrid. Editorial: Paidós.

Troch, A. (1982) El estrés y la personalidad. Barcelona. Editorial: Herder. 